Homology, Homotopy and Applications, vol. 7(3), 2005, pp.173-187

\title{
HIGHER-DIMENSIONAL ARITHMETIC USING $p$-ADIC ÉTALE TATE TWISTS
}

\author{
KANETOMO SATO
}

(communicated by J.F. Jardine)

\begin{abstract}
This paper is a survey on recent researches of the author and his recent joint work with Shuji Saito. We will explain how to construct $p$-adic étale Tate twists on regular arithmetic schemes with semistable reduction, and state some fundamental properties of those objects. We will also explain how to define cycle class maps from Chow groups to étale cohomology groups with coefficients in $p$-adic étale Tate twists and state injectivity and surjectivity results on those new cycle class maps.
\end{abstract}

Dedicated to V. Snaith on his 60 th birthday

\section{Introduction}

In this paper, we like to survey recent results of the author [Sat2], [Sat3] and his joint work with Shuji Saito [SaSa] (cf. [JSS]). Let $k$ be an algebraic number field, i.e., a finite field extension of $\mathbb{Q}$. Let $A$ be the integer ring of $k$. According to the classical unramified class field theory, we have the following isomorphism of finite groups:

$$
\rho_{A}: \operatorname{Cl}(A) \stackrel{\simeq}{\longrightarrow} \pi^{\mathrm{ab}}(\operatorname{Spec}(A))^{\sim},
$$

where $\mathrm{Cl}(A)$ (resp. $\pi^{\mathrm{ab}}(\operatorname{Spec}(A))$ ) denotes the ideal class group of $A$ (resp. étale abelian fundamental group of $\operatorname{Spec}(A))$ and $\sim$ means the quotient group corresponding to abelian coverings of $\operatorname{Spec}(A)$ which splits completely at every real infinite place. This map is defined by sending the class of a prime ideal $\mathfrak{p} \subset A$ to the arithmetic Frobenius substitution of $\operatorname{Spec}(A)$ at $\mathfrak{p}$. By works of Bloch [B1], Kato and Saito $[\mathbf{K S}]$, [Sa1], this isomorphism is generalized to an arbitrary noetherian regular scheme $X$ which is proper flat of finite type over $\operatorname{Spec}(A)$ :

$$
\rho_{X}: \mathrm{CH}_{0}(X) \stackrel{\simeq}{\longrightarrow} \pi^{\mathrm{ab}}(X)^{\sim},
$$

Received October 4, 2004, revised January 7, 2005; published on November 12, 2005.

2000 Mathematics Subject Classification: 14G40, 14F30, 14F42.

Key words and phrases: $p$-adic étale Tate twists, arithmetic duality, unramified cohomology and cycle class maps.

(C) 2005, Kanetomo Sato. Permission to copy for private use granted. 
where $\mathrm{CH}_{0}(X)$ denotes the Chow group of 0-cycles on $X$ modulo rational equivalence. In this paper we like to generalize this map, so called the reciprocity map, to an arbitrary intermediate codimension as an étale cycle class map by introducing $p$-adic étale coefficients which we call $p$-adic étale Tate twists (see (1.1), (4.1) below). We will state some injectivity and surjectivity results on those new cycle class maps (Corollary 4.3, Theorem 5.1). In our injectivity results, the unramified cohomology group $\mathrm{H}_{\mathrm{ur}}^{3}\left(K, \mathbb{Q}_{p} / \mathbb{Z}_{p}(2)\right)$ for the function field $K:=k(X)$ plays a key role, which generalizes the Tate-Shafarevich group of the Jacobian variety of $X_{k}$. We will state finiteness results on this group in $\S 3$, from which we will derive the injectivity results (see (4.2) below). Our surjectivity results, which actually concerns proper smooth or semistable families over local integer rings, is proved by using the arithmetic duality theorem stated in Theorem 2.2 (see $\S 5$ for more details). Using this surjectivity result, we will deduce the triviality of the group $\mathrm{H}_{\mathrm{ur}}^{3}\left(K, \mathbb{Q}_{p} / \mathbb{Z}_{p}(2)\right)$ in some special cases (see Corollary 6.3 below).

\section{Acknowledgements}

The author expresses his gratitude to Professors Victor Snaith and Rick Jardine for giving him this opportunity to write this paper. The researches for this article were supported by JSPS Postdoctoral Fellowship for Research Abroad and EPSRC grant. Thanks are also due to University of Southern California and The University of Nottingham for their great hospitality.

\section{Notation}

For a scheme $X$ and an integer $q \geqslant 0, X^{q}$ denotes the set of all points $x \in X$ of codimension $q$. Unless indicated otherwise, all cohomology groups of schemes are taken over the étale topology.

\section{1. $p$-adic étale Tate twists}

Let $p$ be a rational prime number. Let $A$ be a Dedekind ring whose fraction field has characteristic zero and which has a residue field of characteristic $p$. We always assume that

any residue field of $A$ of characteristic $p$ is perfect.

Let $X$ be a noetherian regular scheme of pure-dimension which is flat of finite type over $B:=\operatorname{Spec}(A)$ and satisfies the following condition:

(*) $X$ is a smooth or semistable family around any fiber of $X / B$ of characteristic $p$.

Let $n$ and $r$ be integers with $n \geqslant 0$ and $r \geqslant 1$.

(1.1) Construction. The $p$-adic étale Tate twist $\mathfrak{T}_{r}(n)_{X}$ is defined as an object $\mathcal{K} \in D^{b}\left(X_{\text {ét }}, \mathbb{Z} / p^{r} \mathbb{Z}\right)$ satisfying the following four properties: 
T1. There exists an isomorphism $t:\left.\mathcal{K}\right|_{V} \simeq \mu_{p^{r}}^{\otimes n}$ with $V:=X \otimes_{A} A[1 / p]$.

T2. $\mathcal{K}$ is concentrated in $[0, n]$.

T3. For a locally closed regular subscheme $i: Z \rightarrow X$ of characteristic $p$, we have a Gysin isomorphism:

$$
W_{r} \Omega_{Z, \log }^{n-c}[-n-c] \stackrel{\simeq}{\longrightarrow} \tau_{\leqslant n+c} R i^{!} \mathcal{K} \quad \text { in } D^{b}\left(Z_{\text {ét }}, \mathbb{Z} / p^{r} \mathbb{Z}\right),
$$

where $c$ denotes $\operatorname{codim}_{X}(Z)$ and $W_{r} \Omega_{Z, \log }^{n-c}$ means the zero sheaf in case $n<c$.

T4. Let $y$ and $x$ be points on $X$ with $\operatorname{ch}(x)=p, x \in \overline{\{y\}}$ and $\operatorname{codim}_{X}(x)=$ $\operatorname{codim}_{X}(y)+1$. Put $c:=\operatorname{codim}_{X}(x)$. Then the boundary map

$$
\delta_{y, x}^{\operatorname{loc}}(\mathcal{K}): \mathrm{H}_{y}^{n+c-1}\left(\operatorname{Spec}\left(\mathcal{O}_{X, y}\right), \mathcal{K}\right) \longrightarrow \mathrm{H}_{x}^{n+c}\left(\operatorname{Spec}\left(\mathcal{O}_{X, x}\right), \mathcal{K}\right)
$$

in the localization theory in étale topology coincides with the following boundary map of Galois cohomology groups defined by Kato ([KCT], §1) via Gysin isomorphisms, up to a sign depending only on $(\operatorname{ch}(y), c)$ :

$$
\partial_{y, x}^{\mathrm{val}}:\left\{\begin{array}{ll}
\mathrm{H}^{n-c+1}\left(y, \mu_{p^{r}}^{\otimes n-c+1}\right) & (\operatorname{ch}(y)=0) \\
\mathrm{H}^{0}\left(y, W_{r} \Omega_{y, \log }^{n-c+1}\right) & (\operatorname{ch}(y)=p)
\end{array}\right\} \longrightarrow \mathrm{H}^{0}\left(x, W_{r} \Omega_{x, \log }^{n-c}\right),
$$

where the Gysin map for $i_{y}$ in case $\operatorname{ch}(y)=0$ arises from the isomorphism $t$ in $\mathbf{T} \mathbf{1}$ and Deligne's cycle class in $\mathrm{H}_{y}^{2 c-2}\left(\operatorname{Spec}\left(\mathcal{O}_{X, y}\right), \mu_{p^{r}}^{\otimes c-1}\right)$.

The properties $\mathbf{T} \mathbf{1}-\mathbf{T} 3$ are $\mathbb{Z} / p^{r} \mathbb{Z}$-coefficient variants of Beilinson-Lichtenbaum axioms on conjectural étale motivic complexes $([\mathbf{B e}],[\mathbf{L i 2}])$. More precisely, T2 is taken from the acyclicity axiom for $\Gamma(n)_{X}^{\text {ét }}$, T1 comes from the axiom of Kummer theory for $\Gamma(n)_{V}^{\text {ét }}$, and T3 is suggested by the purity axiom and the axiom of Kummer theory for $\Gamma(n-c)_{Z}^{\text {ét }}$. Although T4 is not among Beilinson-Lichtenbaum axioms, it is a natural property to be satisfied. In the following theorem, 'unique' means 'unique up to a unique isomorphism' (see [Sat3], Theorem 0.2, Corollary $0.3)$ :

Theorem 1.1. There exists a unique pair $(\mathcal{K}, t)$ of an object $\mathcal{K} \in D^{b}\left(X_{\text {ét }}, \mathbb{Z} / p^{r} \mathbb{Z}\right)$ and an isomorphism $t:\left.\mathcal{K}\right|_{V} \simeq \mu_{p^{r}}^{\otimes n}$ that satisfies $\mathbf{T 2 - T 4}$.

Definition 1.2. For each $n \geqslant 0$ and $r>0$, we fix a pair $(\mathcal{K}, t)$ as in Theorem 1.1 and define $\mathfrak{T}_{r}(n)_{X}:=\mathcal{K}$.

In case $X / B$ is smooth, this object is already considered by Schneider [Sch]. We have $\mathfrak{T}_{r}(0) \simeq \mathbb{Z} / p^{r} \mathbb{Z}$ and $\mathfrak{T}_{r}(n) \simeq R j_{*} \mu_{p^{r}}^{\otimes n}$ for $n>\operatorname{dim}(X)$.

Remark 1.3. Assume that $A$ is local with residue field $\mathbb{F}$ and $p \geqslant n+2$. Let $Y$ be the closed fiber of $X / B$ and let $\iota$ (resp. $j$ ) be the closed (resp. open) immersion $Y \rightarrow X$ (resp. $V \rightarrow X)$.

(1) In case $X / B$ is smooth, $\iota^{*} \mathfrak{T}_{r}(n)_{X}$ is canonically isomorphic to $R \epsilon_{*} S_{r}(n)$ (cf. $[\mathbf{K 1}],[\mathbf{K u}])$, where $S_{r}(n)$ denotes the syntomic complex of Fontaine-Messing $[\mathbf{F M}]$ on the crystalline site $\left(X_{r} / W_{r}\right)_{\text {cris }}$ with $X_{r}:=X \otimes_{A} A / p^{r} A$ and $W_{r}:=$ $W_{r}(\mathbb{F})$, and $\epsilon$ denotes the natural continuous map $\left(X_{r} / W_{r}\right)_{\text {cris }} \rightarrow\left(X_{r}\right)_{\text {ét }}$ of sites. The morphism $t$ corresponds to the Fontaine-Messing morphism $R \epsilon_{*} S_{r}(n) \rightarrow \tau_{\leqslant n} \iota^{*} R j_{*} \mu_{p^{r}}^{\otimes n}$. 
(2) $\iota^{*} \mathfrak{T}_{r}(n)_{X}$ is not the log syntomic complex of Kato and Tsuji $([\mathbf{K 2} \mathbf{2}],[\mathbf{T s} \mathbf{1}])$ unless $n>\operatorname{dim}(X)$, because the latter object is isomorphic to $\tau_{\leqslant n} \iota^{*} R j_{*} \mu_{p^{r}}^{\otimes n}$

by [Ts2]. Therefore $\mathfrak{T}_{r}(n)_{X}$ is a new object particularly on semistable families.

Let $\mathbb{Z}(n)_{X}^{\text {ét }}$ (resp. $\left.\mathbb{Z}(n)_{X}^{Z a r}\right)$ be the étale sheafification (resp. the Zariski sheafification) of Bloch's cycle complex ([B2], [Le1]). By works of Levine ([Le1], [Le2]), these two objects are regarded as strong candidates for the motivic complexes $\Gamma(n)_{X}^{\text {ét }}$ and $\Gamma(n)_{X}^{\mathrm{Zar}}$, respectively, so that we can expect the following conjecture (cf. [Sat3], Conjecture 0.4):

Conjecture 1.4. (1) There exists a canonical isomorphism

$$
\mathbb{Z}(n)_{X}^{\text {ét }} \otimes^{\mathbb{L}} \mathbb{Z} / p^{r} \mathbb{Z} \stackrel{\simeq}{\longrightarrow} \mathfrak{T}_{r}(n)_{X} \quad \text { in } D^{b}\left(X_{\text {ét }}, \mathbb{Z} / p^{r} \mathbb{Z}\right) .
$$

(2) Let $\varepsilon$ be the natural continuous map $X_{\text {ét }} \rightarrow X_{\text {Zar }}$ of sites. Then the morphism in (1) induces an isomorphism

$$
\mathbb{Z}(n)_{X}^{\mathrm{Zar}} \otimes \mathbb{L}^{\mathbb{L}} \mathbb{Z} / p^{r} \mathbb{Z} \stackrel{\simeq}{\longrightarrow} \tau_{\leqslant n} R \varepsilon_{*} \mathfrak{T}_{r}(n)_{X} \quad \text { in } D^{b}\left(X_{\mathrm{Zar}}, \mathbb{Z} / p^{r} \mathbb{Z}\right) .
$$

The case $n=0$ is obvious. The case $n=1$ holds true by the Kummer theory for $\mathbb{G}_{\mathrm{m}}$ (cf. [Sat3], Proposition 3.13):

$$
\mathbb{G}_{\mathrm{m}} \otimes^{\mathbb{L}} \mathbb{Z} / p^{r} \mathbb{Z} \longrightarrow \mathfrak{T}_{r}(1)_{X} \quad \text { in } D^{b}\left(X_{\text {ét }}, \mathbb{Z} / p^{r} \mathbb{Z}\right)
$$

and the isomorphisms $\mathbb{Z}(1)_{X}^{\text {ét }} \simeq \mathbb{G}_{\mathrm{m}}[-1], \mathbb{Z}(1)_{X}^{\text {Zar }} \simeq \varepsilon_{*} \mathbb{G}_{\mathrm{m}}[-1]$ (cf. [Le2], Lemma 11.2 ), and $R^{1} \varepsilon_{*} \mathbb{G}_{\mathrm{m}}=0$ (Hilbert's theorem 90 ). For $n \geqslant 2$, by results of Geisser ([Ge], Theorems $1.2(4), 1.3)$, Conjecture 1.4 (1) holds true in case $X / B$ is smooth, under the Bloch-Kato conjecture on Galois symbol maps.

(1.2) Product structure. The following theorem plays key roles in later sections:

Theorem 1.5. Let $n$ and $n^{\prime}$ be integers at least 0. Then there exists a unique morphism

$$
\mathfrak{T}_{r}(n)_{X} \otimes^{\mathbb{L}} \mathfrak{T}_{r}\left(n^{\prime}\right)_{X} \longrightarrow \mathfrak{T}_{r}\left(n+n^{\prime}\right)_{X} \quad \text { in } D^{-}\left(X_{\text {ét }}, \mathbb{Z} / p^{r} \mathbb{Z}\right)
$$

that extends the natural map $\mu_{p^{r}}^{\otimes n} \otimes \mu_{p^{r}}^{\otimes n^{\prime}} \rightarrow \mu_{p^{r}}^{\otimes n+n^{\prime}}$ on $V_{\text {ét }}$.

Let $Y \subset X$ be the divisor defined by the radical ideal sheaf of $(p) \subset \mathcal{O}_{X}$, and let $\iota$ and $j$ be as follows:

$$
V=X[1 / p] \stackrel{j}{\longrightarrow} X \stackrel{\iota}{\longleftarrow} Y .
$$

To construct the morphism (1.2.1) in case $m \geqslant 1$ and $n \geqslant 1$, the author proved the following natural isomorphism of étale sheaves (cf. [Sat3], Theorem 2.7):

$$
\iota^{*} \mathcal{H}^{n}\left(\mathfrak{T}_{r}(n)_{X}\right) / U^{1} M_{r}^{n} \simeq \operatorname{Im}\left(d \log :\left(\mathbb{G}_{\mathrm{m}, Y}\right)^{\otimes n} \rightarrow \bigoplus_{y \in Y^{0}} \imath_{y *} W_{r} \Omega_{y, \log }^{n}\right)=: \lambda_{Y, r}^{n},
$$

where $U^{\bullet}$ denotes the Bloch-Kato filtration on $M_{r}^{n}:=\iota^{*} R^{n} j_{*} \mu_{p^{r}}^{\otimes n}$ (cf. [Sat3], Definition 2.2) and for $y \in Y, \imath_{y}$ denotes the natural map $y \rightarrow Y$ (in case $Y$ is smooth, we have $\lambda_{Y, r}^{n} \simeq W_{r} \Omega_{Y, \log }^{n}$, and the isomorphism (1.2.2) is due to Bloch-Kato [BK1]). By (1.2.2), it turns out that the sheaf $\mathcal{H}^{n}\left(\mathfrak{T}_{r}(n)_{X}\right)$ is generated by $\iota_{*}\left(U^{1} M_{r}^{n}\right)$ and the image of $\left(\mathbb{G}_{\mathrm{m}}, X\right)^{\otimes n}$, so that the image of the natural cup-product map

$$
\mathcal{H}^{m}\left(\mathfrak{T}_{r}(m)_{X}\right) \otimes \mathcal{H}^{n}\left(\mathfrak{T}_{r}(n)_{X}\right) \longrightarrow R^{m+n} j_{*} \mu_{p^{r}}^{\otimes m+n}
$$


lies in $\mathcal{H}^{m+n}\left(\mathfrak{T}_{r}(m+n)_{X}\right)$. From this fact, we obtain the morphism (1.2.1) as the unique morphism extending the natural map $\mu_{p^{r}}^{\otimes m} \otimes \mu_{p^{r}}^{\otimes n} \rightarrow \mu_{p^{r}}^{\otimes m+n}$ on $V$ (see [Sat3], Proposition 3.3 for details).

Remark 1.6. For $n \geqslant 0$, let $\nu_{Y, r}^{n}$ be the kernel of the following boundary map of logarithmic Hodge-Witt sheaves:

$$
\bigoplus_{y \in Y^{0}} \imath_{y *} W_{r} \Omega_{y, \log }^{n} \longrightarrow \bigoplus_{x \in Y^{1}} \imath_{x *} W_{r} \Omega_{x, \log }^{n-1},
$$

where for $x \in Y, \imath_{x}: x \rightarrow Y$ is as defined above. Then one can easily see that we have $\lambda_{Y, r}^{n} \subset \nu_{Y, r}^{n}$ in general, and that $\lambda_{Y, r}^{n}=\nu_{Y, r}^{n}=W_{r} \Omega_{Y, \log }^{n}$ in case $Y$ is smooth. In case $Y$ has normal crossings, the fundamental properties of $\nu_{Y, r}^{n}$ and $\lambda_{Y, r}^{n}$ are studied in [Sat2]. Moreover, we have the following isomorphism for $n \geqslant 1$ :

$$
M_{r}^{n} / \iota^{*} \mathcal{H}^{n}\left(\mathfrak{T}_{r}(n)_{X}\right) \simeq \nu_{Y, r}^{n-1}
$$

(cf. [Sat3], Theorem 2.7; compare with (1.2.2)).

\section{Arithmetic duality theorems}

Let $A$ be either a global integer ring or a $p$-adic local integer ring, and put $B:=$ $\operatorname{Spec}(A)$. Let $p$ and $X / B$ be as in $\S 1$. In case $A$ is global, for $\mathcal{L} \in D^{+}\left(X_{\text {ét }}, \mathbb{Z} / p^{r} \mathbb{Z}\right)$, we define the $m$ th étale cohomology group $\mathrm{H}_{c}^{m}(X, \mathcal{L})$ with compact support to be $\mathrm{H}_{c}^{m}\left(B, R f_{!} \mathcal{L}\right)$, where $f$ denotes the structure morphism $X \rightarrow B$ and $\mathrm{H}_{c}^{*}(B, \bullet)$ denotes the étale cohomology group with compact support of $B$ (cf. [Mi2], II.2). In this section, we give the statements and an outline of the duality results that the author proved in [Sat3], $\S 6$ (see also [JSS] for other duality results).

(2.1) Duality theorems. We first assume that $A$ is global. For $V=X[1 / p]$, we have the following well-known pairing with $d:=\operatorname{dim}(X)$, the absolute dimension of $X:$

$$
\mathrm{H}_{c}^{i}\left(V, \mu_{p^{r}}^{\otimes n}\right) \times \mathrm{H}^{2 d+1-i}\left(V, \mu_{p^{r}}^{\otimes d-n}\right) \longrightarrow \mathrm{H}_{c}^{2 d+1}\left(V, \mu_{p^{r}}^{\otimes d}\right) \stackrel{\operatorname{tr}_{V}}{\longrightarrow} \mathbb{Z} / p^{r} \mathbb{Z},
$$

which is a non-degenerate pairing of finite groups by the Artin-Verdier duality ([AV], $[\mathbf{M a}],[\mathbf{M i 2}]$; see also $[\mathbf{D n}],[\mathbf{S p}])$ and the relative Poincaré duality for regular schemes ([SGA4], XVIII, [Th], [Fu]).

Theorem 2.1. Assume that $A$ is global and that $X$ is proper over $B$. Then:

(1) There exists a canonical trace map $\operatorname{tr}_{X}: \mathrm{H}_{c}^{2 d+1}\left(X, \mathfrak{T}_{r}(d)_{X}\right) \rightarrow \mathbb{Z} / p^{r} \mathbb{Z}$ extending $\operatorname{tr}_{V}$. If $X$ is connected, then $\operatorname{tr}_{X}$ is bijective.

(2) For any integers $n$ and $i$ with $0 \leqslant n \leqslant d$, the natural pairing

$$
\mathrm{H}_{c}^{i}\left(X, \mathfrak{T}_{r}(n)_{X}\right) \times \mathrm{H}^{2 d+1-i}\left(X, \mathfrak{T}_{r}(d-n)_{X}\right) \longrightarrow \mathbb{Z} / p^{r} \mathbb{Z},
$$

arising from (1.2.1) and $\operatorname{tr}_{X}$, is a non-degenerate pairing of finite $\mathbb{Z} / p^{r} \mathbb{Z}$ modules.

By the non-degeneracy of the above pairing for $V$, Theorem 2.1 is reduced to the following duality theorem: 
Theorem 2.2. Assume that $A$ is local and that $X$ is proper over $B$. Then:

(1) There exists a canonical trace map $\operatorname{tr}_{(X, Y)}: \mathrm{H}_{Y}^{2 d+1}\left(X, \mathfrak{T}_{r}(d)_{X}\right) \rightarrow \mathbb{Z} / p^{r} \mathbb{Z}$, which is bijective if $X$ is connected.

(2) For any integers $n$ and $i$ with $0 \leqslant n \leqslant d$, the natural pairing

$$
\mathrm{H}_{Y}^{i}\left(X, \mathfrak{T}_{r}(n)_{X}\right) \times \mathrm{H}^{2 d+1-i}\left(X, \mathfrak{T}_{r}(d-n)_{X}\right) \longrightarrow \mathbb{Z} / p^{r} \mathbb{Z},
$$

arising from (1.2.1) and $\operatorname{tr}_{(X, Y)}$, is a non-degenerate pairing of finite $\mathbb{Z} / p^{r} \mathbb{Z}$ modules.

(2.2) Outline of Theorem 2.2. Without loss of generality, we may assume that $X$ is integral and $A=\Gamma\left(X, \mathcal{O}_{X}\right)$. Let $Y$ be the closed fiber of $X / B$. By a standard argument using the Bockstein triangle (cf. [Sat3], Proposition 3.5)

$$
\mathfrak{T}_{r}(n)_{X} \longrightarrow \mathfrak{T}_{1}(n)_{X} \stackrel{\delta_{1, r-1}}{\longrightarrow} \mathfrak{T}_{r-1}(n)_{X}[1] \longrightarrow \mathfrak{T}_{r}(n)_{X}[1],
$$

we are reduced to the case $r=1$, and then by a standard norm argument we are further reduced to the case where $\operatorname{Frac}(A)$ contains primitive $p$ th roots of unity. By the isomorphisms (1.2.2) and (1.2.3), the non-degeneracy of (2.1.2) is reduced to that of the following two pairings:

$$
\begin{gathered}
\mathrm{H}^{i}\left(Y, \nu_{Y, 1}^{t}\right) \times \mathrm{H}^{d-i}\left(Y, \lambda_{Y, 1}^{d-1-t}\right) \longrightarrow \mathbb{Z} / p \mathbb{Z}, \\
\mathrm{H}^{i}\left(Y, U^{1} M_{1}^{t}\right) \times \mathrm{H}^{d-1-i}\left(Y, U^{1} M_{1}^{d+1-t}\right) \longrightarrow \mu,
\end{gathered}
$$

where $\mu$ denotes the group of $p$ th roots of unity in $\operatorname{Frac}(A)$; we need to consider several t's to prove Theorem 2.2 for a single $n$. The non-degeneracy of $(2.2 .1)$ is due to Milne [Mi1] in case $Y$ is smooth, and proved by the author [Sat2] in case $Y$ has normal crossings. The most delicate part of our duality is the non-degeneracy of (2.2.2). In fact, this pairing arises from the following morphism obtained by decomposing the morphism (1.2.1) with $(m, n)=(t, d+1-t)$ :

$$
U^{1} M_{1}^{t} \otimes U^{1} M_{1}^{d+1-t} \longrightarrow\left(\iota^{*} j_{*} \mu_{p} \otimes \nu_{Y, 1}^{d-1}\right)[1] \quad \text { in } \quad D^{b}\left(Y_{\text {ét }}, \mathbb{Z} / p \mathbb{Z}\right),
$$

where $M_{1}^{m}$ and $U^{\bullet}$ are as in (1.2). The author proved an explicit formula (cf. [Sat3], Theorem 5.6) to compute this morphism in terms of products of symbols and a simple connecting morphism in $D^{b}\left(Y_{\text {ét }}, \mathbb{Z} / p \mathbb{Z}\right)$. By that formula, the nondegeneracy of (2.2.2) is reduced to the Serre-Hartshorne duality for cohomology groups of $Y$ with coefficients in differential modules.

\section{Unramified cohomology and finiteness}

In this and the later sections, we work with the following setting. Let $k$ be either a local field or a global field. Let $A$ be the integer ring of $k$. Let $p$ be a prime number, and let $X$ be a noetherian regular scheme which is proper flat of finite type over $B:=\operatorname{Spec}(A)$. In case $p$ is not invertible in $A$ we always assume that $X$ has good or semistable reduction at all closed points on $B$ of characteristic $p$. In case $p$ is 
invertible in $A$ (i.e., $k$ is $\ell$-adic local with $\ell \neq p), \mathfrak{T}_{r}(n)_{X}$ means the usual Tate twist $\mu_{p^{r}}^{\otimes n}$. Let $K$ be the function field of $X$. We will often write $X_{k}$ for $X \otimes_{A} k$.

(3.1) Unramified cohomology. We define the unramified cohomology group $\mathrm{H}_{\mathrm{ur}}^{n+1}\left(K, \mathbb{Q}_{p} / \mathbb{Z}_{p}(n)\right)$ as the kernel of the boundary map

$\mathrm{H}^{n+1}\left(\operatorname{Spec}(K), \mathbb{Q}_{p} / \mathbb{Z}_{p}(n)\right) \longrightarrow \bigoplus_{y \in X^{1}} \mathrm{H}_{y}^{n+2}\left(\operatorname{Spec}\left(\mathcal{O}_{X, y}\right),\left.\mathfrak{T}_{\infty}(n)_{X}\right|_{\operatorname{Spec}\left(\mathcal{O}_{X, y}\right)}\right)$,

where $\mathfrak{T}_{\infty}(n)_{X}$ denotes $\lim _{r \geqslant 1} \mathfrak{T}_{r}(n)_{X}$. The $y$-component of the group on the right hand side is isomorphic to $\mathrm{H}^{n}\left(y, \mathbb{Q}_{p} / \mathbb{Z}_{p}(n-1)\right)$, if $\operatorname{ch}(y) \neq p$. The following isomorphisms hold true:

$$
\mathrm{H}_{\mathrm{ur}}^{1}\left(K, \mathbb{Q}_{p} / \mathbb{Z}_{p}(0)\right) \simeq \mathrm{H}^{1}\left(X, \mathbb{Q}_{p} / \mathbb{Z}_{p}\right) \text { and } \mathrm{H}_{\mathrm{ur}}^{2}\left(K, \mathbb{Q}_{p} / \mathbb{Z}_{p}(1)\right) \simeq \operatorname{Br}(X)_{p \text {-tors }},
$$

where $\operatorname{Br}(X)$ denotes the Grothendieck-Brauer group $\mathrm{H}^{2}\left(X, \mathbb{G}_{\mathrm{m}}\right)$ (see [SaSa]). An intriguing question is whether $\mathrm{H}_{\mathrm{ur}}^{n+1}\left(K, \mathbb{Q}_{p} / \mathbb{Z}_{p}(n)\right)$ is finite. In fact, this question is related to several significant theorems and conjectures in arithmetic geometry (see Remark 3.1 below). Moreover, the finiteness of $\mathrm{H}_{\mathrm{ur}}^{3}\left(K, \mathbb{Q}_{p} / \mathbb{Z}_{p}(2)\right)$ and its subgroup

$$
\begin{aligned}
& \mathrm{H}_{\mathrm{ur}}^{3}\left(K, X_{k} ; \mathbb{Q}_{p} / \mathbb{Z}_{p}(2)\right):= \\
& \quad \operatorname{Im}\left(\mathrm{H}^{3}\left(X_{k}, \mathbb{Q}_{p} / \mathbb{Z}_{p}(2)\right) \rightarrow \mathrm{H}^{3}\left(\operatorname{Spec}(K), \mathbb{Q}_{p} / \mathbb{Z}_{p}(2)\right)\right) \cap \mathrm{H}_{\mathrm{ur}}^{3}\left(K, \mathbb{Q}_{p} / \mathbb{Z}_{p}(2)\right)
\end{aligned}
$$

implies the injectivity of the cycle class map of $X$ of codimension 2 and its torsion part, respectively (see $\S 4$ below).

Remark 3.1. (1) For $n=0$, the quotient $\mathrm{H}^{1}(X, \mathbb{Q} / \mathbb{Z}) / \mathrm{H}^{1}(B, \mathbb{Q} / \mathbb{Z})$ is finite by a theorem of Katz-Lang $[\mathbf{K L}]$; in case $k$ is global, $\mathrm{H}_{\mathrm{ur}}^{1}\left(K, \mathbb{Q}_{p} / \mathbb{Z}_{p}(0)\right)$ is finite as well, because $\mathrm{H}^{1}(B, \mathbb{Q} / \mathbb{Z})$ is finite.

(2) In case $n=1$ and $k$ is global, the finiteness of $\mathrm{H}_{\mathrm{ur}}^{2}\left(K, \mathbb{Q}_{p} / \mathbb{Z}_{p}(1)\right)$ is equivalent to the finiteness of $\operatorname{Br}(X)_{p \text {-tors }}$.

(3) For $n=d:=\operatorname{dim}(X), \mathrm{H}_{\mathrm{ur}}^{d+1}\left(K, \mathbb{Q}_{p} / \mathbb{Z}_{p}(d)\right)$ is a group considered by Kato $[$ KCT $]$. He conjectured that $\mathrm{H}_{\mathrm{ur}}^{d+1}\left(K, \mathbb{Q}_{p} / \mathbb{Z}_{p}(d)\right)=0$ if $p \neq 2$ or $k$ has no embedding into $\mathbb{R}$. This conjecture is viewed as a generalization of the classical Hasse principle on the Brauer groups of global and local fields. The case $d=2$ is proved in $[\mathbf{K C T}]$, and the case $d=3$ is proved in $[\mathbf{J S}]$.

(3.2) Finiteness. We state here the following results proved in [SaSa] (cf. loc. cit., Theorem 0.3, Corollary 0.4), where $\kappa_{X_{k}}$ denotes the Kodaira dimension of $X_{k}$ :

Theorem 3.2. Assume $\mathrm{H}^{2}\left(X_{k}, \mathcal{O}_{X_{k}}\right)=0$. Then:

(1) $\mathrm{H}_{\mathrm{ur}}^{3}\left(K, X_{k} ; \mathbb{Q}_{p} / \mathbb{Z}_{p}(2)\right)$ is finite.

(2) If $k$ is $\ell$-adic local with $\ell \neq p$ and $X_{k}$ is a surface, then $\mathrm{H}_{\mathrm{ur}}^{3}\left(K, \mathbb{Q}_{p} / \mathbb{Z}_{p}(2)\right)$ is finite.

(3) If $k$ is $p$-adic local and $X_{k}$ is a surface with $\kappa_{X_{k}} \leqslant 1$, then $\mathrm{H}_{\mathrm{ur}}^{3}\left(K, \mathbb{Q}_{p} / \mathbb{Z}_{p}(2)\right)$ is finite.

(4) If $k$ is global and $X_{k}$ is a surface with $\kappa_{X_{k}} \leqslant 1$, then $\mathrm{H}_{\mathrm{ur}}^{3}\left(K, \mathbb{Q}_{p} / \mathbb{Z}_{p}(2)\right)$ is finite. 
The conditions in this theorem comes from those in results of Bloch-Kas-Lieberman $[\mathbf{B K L}]$, Salberger [Sal], Colliot-Thélène-Raskind [CTR] and Saito-Sujatha [SaSu]. More precisely, we use $p$-adic Hodge Theory $([\mathbf{K 2}]$, [Ts1]) and arguments of the author in [Sat1], to reduce the problem to the above well-known facts. In case $k$ is global, we need a local-global principle due to Jannsen ([J] $] \S 4$, Theorem 4).

Remark 3.3. It would be a serious problem to prove the above finiteness theorems unconditionally (cf. $[\mathbf{L S}],[\mathbf{L a} 2],[\mathbf{O}])$. In fact, the finiteness of $\mathrm{H}_{\mathrm{ur}}^{3}\left(K, X_{k} ; \mathbb{Q}_{p} / \mathbb{Z}_{p}(2)\right)$ and $\mathrm{H}_{\mathrm{ur}}^{3}\left(K, \mathbb{Q}_{p} / \mathbb{Z}_{p}(2)\right)$ in the general case is deduced from several well-known conjectures in arithmetic geometry which are known to hold true in the situations in Theorem 3.2. See [SaSa], (0.1) for precise statements and loc. cit., Chapter I for detailed proofs.

\section{Cycle class maps and injectivity in codimension 2}

Let the notation be as in $\S 3$. We state here the definition of cycle class maps and the injectivity results on the cycle class map of $X$ of codimension 2 proved in $[\mathbf{S a S a}]$, Chapter I, which is deduced from the finiteness theorems mentioned in $(3.2)$.

(4.1) Definition of cycle class maps. We define the cycle class map

$$
\rho_{X, p^{r}}^{n}: \mathrm{CH}^{n}(X) / p^{r} \longrightarrow \mathrm{H}^{2 n}\left(X, \mathfrak{T}_{r}(n)_{X}\right)
$$

as follows. We have the localization spectral sequence

$$
E_{1}^{u, v}=\bigoplus_{x \in X^{u}} \mathrm{H}_{x}^{u+v}\left(X, \mathfrak{T}_{r}(n)_{X}\right) \Longrightarrow \mathrm{H}^{u+v}\left(X, \mathfrak{T}_{r}(n)_{X}\right) .
$$

By the purity property T3 in (1.1) and the absolute cohomological purity (cf. [Th], $[\mathbf{F u}])$, we have

$$
E_{1}^{u, v} \simeq \bigoplus_{x \in X^{u}} \mathrm{H}^{v-u}\left(x, \mathbb{Z} / p^{r} \mathbb{Z}(n-u)_{x}\right), \quad \text { if } v \leqslant n .
$$

This implies that we have the edge homomorphism $E_{2}^{n, n} \rightarrow \mathrm{H}^{2 n}\left(X, \mathfrak{T}_{r}(n)_{X}\right)$ with

$$
\begin{aligned}
E_{2}^{n, n} & \simeq \operatorname{Coker}\left(\bigoplus_{y \in X^{n-1}} \mathrm{H}^{1}\left(y, \mathbb{Z} / p^{r} \mathbb{Z}(1)_{y}\right) \stackrel{\partial^{\mathrm{val}}}{\longrightarrow} \bigoplus_{x \in X^{n}} \mathrm{H}^{0}\left(x, \mathbb{Z} / p^{r} \mathbb{Z}\right)\right) \\
& \simeq \mathrm{CH}^{n}(X) / p^{r},
\end{aligned}
$$

where $\partial^{\text {val }}$ is the sum of boundary maps $\partial_{y, x}^{\text {val }}$ with $y \in X^{n-1}$ and $x \in X^{n}$ (cf. T4 in (1.1)). Thus we obtain the map $\rho_{X, p^{r}}^{n}$ and the following induced maps:

$$
\begin{aligned}
\rho_{X, p \text {-tors }, r}^{n}: & \mathrm{CH}^{n}(X)_{p \text {-tors }} \longrightarrow \mathrm{H}^{2 n}\left(X, \mathfrak{T}_{r}(n)_{X}\right), \\
\rho_{X, \mathbb{Z}_{p}}^{n}: & \mathrm{CH}^{n}(X) \otimes \mathbb{Z}_{p} \longrightarrow \mathrm{H}^{2 n}\left(X, \mathfrak{T}_{\mathbb{Z}_{p}}(n)_{X}\right),
\end{aligned}
$$

where for an abelian group $M, M_{p \text {-tors }}$ denotes the $p$-primary torsion part, and $\mathrm{H}^{*}\left(X, \mathfrak{T}_{\mathbb{Z}_{p}}(n)_{X}\right)$ is defined as $\lim _{r \geqslant 1} \mathrm{H}^{*}\left(X, \mathfrak{T}_{r}(n)_{X}\right)$.

Remark 4.1. In case $A$ is global and $X / B$ is proper, the map $\rho_{X, \mathbb{Z}_{p}}^{d}$ with $d:=\operatorname{dim}(X)$ coincides with the $p$-adic part of the reciprocity map $\rho_{X}$ in the introduction (cf. Theorem 2.1). 
(4.2) Injectivity in codimension 2 . For an abelian group $M$, let $M_{\text {Div }}\left(\operatorname{resp} . M_{p \text {-Div }}\right)$ be the maximal divisible subgroup (resp. the maximal $p$-divisible subgroup). In the rest of this section, we restrict our attention to the case $n=2$. In this case, we have the following proposition, which reduces the injectivity of cycle class maps to the finiteness of unramified cohomology groups (cf. [SaSa], Proposition 0.2):

Proposition 4.2. (1) Assume that $\mathrm{H}_{\mathrm{ur}}^{3}\left(K, X_{k} ; \mathbb{Q}_{p} / \mathbb{Z}_{p}(2)\right)$ is finite. Then there exists a positive integer $r_{0}$ such that $\operatorname{Ker}\left(\rho_{X, p \text {-tors }, r}^{2}\right)=\left(\mathrm{CH}^{2}(X)_{p \text {-tors }}\right)_{\text {Div }}$ for any $r \geqslant r_{0}$.

(2) Assume that $\mathrm{H}_{\mathrm{ur}}^{3}\left(K, \mathbb{Q}_{p} / \mathbb{Z}_{p}(2)\right)$ is finite. Then we have

$$
\operatorname{Ker}\left(\rho_{X, \mathbb{Z}_{p}}^{2}\right)=\left(\mathrm{CH}^{2}(X) \otimes \mathbb{Z}_{p}\right)_{\text {Div }},
$$

and the kernel of the map

$$
\rho^{\prime}: \mathrm{CH}^{2}(X) \longrightarrow \mathrm{H}^{4}\left(X, \mathfrak{T}_{\mathbb{Z}_{p}}(2)_{X}\right)
$$

induced by $\rho_{X, \mathbb{Z}_{p}}^{2}$ coincides with $\mathrm{CH}^{2}(X)_{p \text {-Div }}$.

This proposition is proved by standard computations on the spectral sequence (4.1.1) together with a version of the commutative diagram of Colliot-ThélèneSansuc-Soulé in [CTSS], §1 (cf. [SaSa] Chapter I, Lemma 3.4). By Theorem 3.2 and Proposition 4.2, we obtain the following corollary:

Corollary 4.3. Assume $\mathrm{H}^{2}\left(X_{k}, \mathcal{O}_{X_{k}}\right)=0$. Then:

(1) $\mathrm{CH}^{2}(X)_{p \text {-tors }}$ is finite and $\rho_{X, p \text {-tors, } r}^{2}$ is injective for a sufficiently large $r$.

(2) If $k$ is $\ell$-adic local with $\ell \neq p$ and $X_{k}$ is a surface, then $\operatorname{Ker}\left(\rho_{X, \mathbb{Z}_{p}}^{2}\right)$ and $\operatorname{Ker}\left(\rho^{\prime}\right)$ are uniquely $p$-divisible.

(3) If $k$ is $p$-adic local, and $X_{k}$ is a surface with $\kappa_{X_{k}} \leqslant 1$, then $\rho^{\prime}$ is injective up to prime-to-p torsion elements.

(4) If $k$ is global and $X_{k}$ is a surface with $\kappa_{X_{k}} \leqslant 1$, then $\rho_{X, \mathbb{Z}_{p}}^{2}$ is injective.

The finiteness part of Corollary 4.3 (1) is originally due to Salberger [Sal], ColliotThélène and Raskind [CTR].

Remark 4.4. If $\mathrm{H}^{2}\left(X_{k}, \mathcal{O}_{X_{k}}\right)=0$ and the Albanese variety of $X_{k}$ has potentially good reduction at every closed point on $B$, then the torsion cycle class map $\rho_{X_{k}, p \text {-tors }, r}^{2}$ of $X_{k}$ of codimension 2 is injective for a sufficiently large $r>0$ [Sa4]. Moreover, if the Albanese variety does not have potentially good reduction, then we cannot expect the same injectivity any longer in general $[\mathbf{P S}],[\mathbf{S u}]$. However Corollary 4.3 shows that we recover satisfactory injectivity properties by considering the cycle class map of $X$ (not of $X_{k}$ ).

\section{Surjectivity of 1-cycle class map}

Let the notation be as in $\S 3$. We explain here the surjectivity result on 1-cycle class maps proved in $[\mathbf{S a S a}]$, Chapter II, $\S 5$. Let $d$ be the absolute dimension of $X$. 
Theorem 5.1. Assume that $k$ is local. Then $\rho_{X, p^{r}}^{d-1}$ is surjective for any $r \geqslant 1$.

Note that this theorem is unconditional on $X$. We show an outline of a proof of Theorem 5.1. Let $Y \subset X$ be the closed fiber of $X / B$ and take a dense open regular subset $U$ of the reduced part of $Y$. Let $\ell$ be the residual characteristic of $k$. For $x \in X$, let $A_{x}$ be the henselization of $\mathcal{O}_{X, x}$ at its maximal ideal. For a scheme $Z$, let $\operatorname{Br}(Z)$ be the cohomological Brauer group $\mathrm{H}^{2}\left(Z, \mathbb{G}_{\mathrm{m}}\right)$. Let $\left(X_{k}\right)_{0}$ and $(Y)_{0}$ be the sets of all closed points on $X_{k}$ and $Y$, respectively, and let sp : $\left(X_{k}\right)_{0} \rightarrow(Y)_{0}$ be the specialization map of points. By Theorem 2.2, we have the natural isomorphism

$$
a: \mathrm{H}^{2 d-2}\left(X, \mathfrak{T}_{r}(d-1)_{X}\right) \stackrel{\simeq}{\longrightarrow} \mathrm{H}_{Y}^{3}\left(X, \mathfrak{T}_{r}(1)_{X}\right)^{*},
$$

where we put $M^{*}:=\operatorname{Hom}(M, \mathbb{Q} / \mathbb{Z})$ for an abelian group $M$. A key step of our proof is to construct an injective map

$$
\theta_{p^{r}}: \mathrm{H}_{Y}^{3}\left(X, \mathfrak{T}_{r}(1)_{X}\right) \longrightarrow \prod_{x \in(U)_{0} p^{r}} \operatorname{Br}\left(A_{x}[1 / \ell]\right)
$$

whose dual fits into the following commutative diagram:

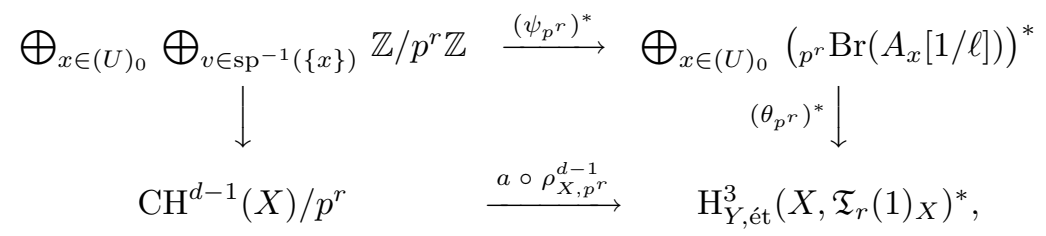

where $\left(\psi_{p^{r}}\right)^{*}$ is the direct sum of the dual of the following map for $x \in(U)_{0}$ :

$$
\psi_{x, p^{r}}: p^{r} \operatorname{Br}\left(A_{x}[1 / \ell]\right) \longrightarrow \prod_{v \in \mathrm{sp}^{-1}(\{x\})} \mathbb{Z} / p^{r} \mathbb{Z} ; \quad \omega \mapsto\left(\operatorname{inv}_{v}\left(\left.\omega\right|_{v}\right)\right)_{v}
$$

and $\operatorname{inv}_{v}$ denotes the invariant isomorphism ${ }_{p^{r}} \operatorname{Br}(v) \simeq \mathbb{Z} / p^{r} \mathbb{Z}$. More precisely, $\theta_{p^{r}}$ is defined as a natural restriction map and the commutativity of the above diagram is proved by a projection formula for $p$-adic étale Tate twists (cf. [Sat3], Corollary 4.10). By the above commutative diagram and the surjectivity of $\left(\theta_{p^{r}}\right)^{*}$, Theorem 5.1 is reduced to the following local result:

Theorem 5.2. $\psi_{x, p^{r}}$ is injective for any $x \in(U)_{0}$ and $r \geqslant 1$.

This theorem is proved by an induction argument on the dimension of local rings and the Brauer group theory of 2-dimensional local rings due to Saito [Sa2] (see [SaSa], Chapter II, $\S 1, \S 2$ for details).

Remark 5.3. Theorem 5.2 has an application to the Brauer-Manin pairing

$$
\langle,\rangle: \mathrm{CH}_{0}\left(X_{k}\right) \times \operatorname{Br}\left(X_{k}\right) \rightarrow \mathbb{Q} / \mathbb{Z} .
$$

In fact, using Theorem 5.2, we proved the $p$-primary variant of results of ColliotThélène and Saito [CTS] (see [SaSa], Chapter II, $\S 3, \S 4$ ).

As a direct consequence of Corollary 4.3 (2) and Theorem 5.1, we obtain

Corollary 5.4. Assume that $k$ is p-adic local and that $X_{k}$ is a surface with $\mathrm{H}^{2}\left(X_{k}, \mathcal{O}_{X_{k}}\right)=0$ and $\kappa_{X_{k}} \leqslant 1$. Then the cycle class map

$$
\mathrm{CH}^{2}(X) \longrightarrow \mathrm{H}^{4}\left(X, \mathfrak{T}_{\mathbb{Z}_{p}}(2)_{X}\right) \times \prod_{\ell \neq p} \mathrm{H}^{4}\left(X, \mathbb{Z}_{\ell}(2)\right)
$$


is injective and has dense image with respect to the pro-finite topology on the group on the right hand side.

See [Ya] for a comparison fact between formal $K$-cohomology groups and syntomic cohomology groups.

Remark 5.5. In case $k$ is global, the surjectivity of $\rho_{X, p^{r}}^{d-1}$ implies the $p$-adic part of the Hasse principle for 0-cycles on $X_{k}$ up to the Brauer-Manin obstruction (cf. [Sa3], [CT1], [CT2], [SaSa], Theorem 0.13).

\section{Triviality of unramified cohomology}

The notation remains as in $\S 5$; we assume that $k$ is local. In this section, we like to mention some consequences of Theorem 3.2 and Theorem 5.1. We first recall the following fact proved in $[\mathbf{S a S a}]$, Chapter I, Proposition 3.7:

Proposition 6.1. Assume that $\mathrm{H}_{\mathrm{ur}}^{3}\left(K, \mathbb{Q}_{p} / \mathbb{Z}_{p}(2)\right)$ is finite. Then the order of

$$
\operatorname{Coker}\left(\rho_{X, \mathbb{Z}_{p}}^{2}: \mathrm{CH}^{2}(X) \otimes \mathbb{Z}_{p} \rightarrow \mathrm{H}^{4}\left(X, \mathfrak{T}_{\mathbb{Z}_{p}}(2)_{X}\right)\right)_{\text {tors }}
$$

coincides with that of $\mathrm{H}_{\mathrm{ur}}^{3}\left(K, \mathbb{Q}_{p} / \mathbb{Z}_{p}(2)\right)$.

This proposition is proved by standard arguments using the spectral sequence (4.1.1), and gives a generalization of the isomorphism

$$
\mathrm{H}_{\mathrm{ur}}^{2}\left(K, \mathbb{Q}_{p} / \mathbb{Z}_{p}(1)\right) \simeq \operatorname{Br}(X)_{p \text {-tors }}
$$

By Proposition 6.1 and Theorem 5.1, we obtain

Corollary 6.2. Assume that $d=3$ (i.e., $X_{k}$ is a surface). Then $\mathrm{H}_{\mathrm{ur}}^{3}\left(K, \mathbb{Q}_{p} / \mathbb{Z}_{p}(2)\right)$ is finite if and only if it is trivial.

Hence by Theorem 3.2, we obtain the following corollary, which generalizes a result of Spiess $([\mathbf{S p}], \S 4)$ to more general situations:

Corollary 6.3. Assume that $d=3$ and that $\mathrm{H}^{2}\left(X_{k}, \mathcal{O}_{X_{k}}\right)=0$.

(1) If $k$ is $\ell$-adic local with $\ell \neq p$, then we have $\mathrm{H}_{\mathrm{ur}}^{3}\left(K, \mathbb{Q}_{p} / \mathbb{Z}_{p}(2)\right)=0$.

(2) If $k$ is $p$-adic local and $\kappa_{X_{k}} \leqslant 1$, then we have $\mathrm{H}_{\mathrm{ur}}^{3}\left(K, \mathbb{Q}_{p} / \mathbb{Z}_{p}(2)\right)=0$.

\section{References}

[AV] Aritin, M., Verdier, J.-L.: Seminar on étale cohomology of number fields. Woods Hole, 1964

[Be] Beilinson, A. A.: Height pairings between algebraic cycles. In: Manin, Yu. I. (ed.) K-theory, Arithmetic and Geometry, (Lecture Notes in Math. 1289), pp. 1-27, Berlin, Springer, 1987 
[BBD] Beilinson, A. A., Bernstein, J., Deligne, P.: Faisceaux Pervers. (Astérisque 100), Soc. Math. France, 1982

[B1] Bloch, S.: Algebraic $K$-theory and classfield theory for arithmetic surfaces. Ann. of Math. 114, 229-265 (1981)

[B2] Bloch, S.: Algebraic cycles and higher $K$-theory. Adv. Math. 61, 267304 (1986)

[BKL] Bloch, S., Kas, A., Lieberman, D.: Zero cycles on surfaces with $p_{g}=0$. Compositio Math. 33, 135-145 (1976)

[BK1] Bloch, S., Kato, K.: p-adic étale cohomology. Inst. Hautes Études Sci. Publ. Math. 63, 107-152 (1986)

[CT1] Colliot-Thélène, J.-L.: L'arithmétique du groupede Chow des zérocycles. J. Théor. Nombres Bordeaux 7, 51-73 (1995)

[CT2] Colliot-Thélène, J.-L.: Conjectures de type local-global sur l'image des groupes de Chow dans la cohomologie étale. In: Algebraic K-theory, Seattle, 1997, (Proc. of Sympos. Pure Math. 67), pp. 1-12, Providence, Amer. Math. Soc., 1999

[CTR] Colliot-Thélène, J.-L., Raskind, W.: Groupe de Chow de codimension deux des variété sur un corps de numbres: Un théorème de finitude pour la torsion, Invent. Math. 105, 221-245 (1991)

[CTS] Colliot-Thélène, J.-L., Saito, S.: Zéro-cycles sur les variétés p-adiques et groupe de Brauer. Internat. Math. Res. Notices 1996, 151-160

[CTSS] Colliot-Thélène, J.-L., Sansuc, J.-J., Soulé, C.: Torsion dans le groupe de Chow de codimension deux. Duke Math. J. 50, 763-801 (1983)

[Dn] Deninger, C.: Duality in the étale cohomology of one-dimensional schemes and generalizations. Math. Ann. 277, 529-541 (1987)

[FM] Fontaine, J.-M., Messing, W.: $p$-adic periods and $p$-adic étale cohomology. In: Ribet, K. A. (ed.) Current Trends in Arithmetical Algebraic Geometry, (Contemp. Math. 67), pp. 179-207, Providence, Amer. Math. Soc., 1987

[Fu] Fujiwara, K.: A proof of the absolute purity conjecture (after Gabber). In: Usui, S., Green, M., Illusie, L., Kato, K., Looijenga, E., Mukai, S., Saito, S. (eds.) Algebraic Geometry, Azumino, 2001, (Adv. Stud. in Pure Math. 36), pp. 153-184, Tokyo, Math. Soc. Japan, 2002

[Ge] Geisser, T.: Motivic cohomology over Dedekind rings. preprint, 2001

[Gr1] Gros, M.: Classes de Chern et classes des cycles en cohomologie logarithmique. Bull. Soc. Math. France Mémoire No 21, 1985

[Gr2] Gros, M.: Sur la partie $p$-primaire du groupe de Chow de codimension deux. Comm. Algebra 13, 2407-2420 (1985)

[Ha] Hagihara, K.: Application of $p$-adic Hodge theory to the coniveau filtration. Master's thesis, University of Tokyo, 1999.

[Hy] Hyodo, O.: A note on $p$-adic etale cohomology in the semi-stable reduction case. Invent. Math. 91 543-557 (1988) 
[J] Jannsen, U.: On the $\ell$-adic cohomology of varieties over number fields and its Galois cohomology. In: Ihara, Y., Ribet, K. A., Serre, J.-P. (eds.) Galois Group over $\mathbb{Q}$, pp. 315-360, Berlin, Springer, 1989

[JS] Jannsen, U., Saito, S.: Kato homology of arithmetic schemes and higher class field theory over local fields. Documenta Math. Extra Volume: Kazuya Kato's Fiftieth Birthday, 479-538 (2003)

[JSS] Jannsen, U., Saito, S., Sato, K.: Étale duality for constructible sheaves on arithmetic schemes (preliminary version). preprint

[K1] Kato, K.: On $p$-adic vanishing cycles (Application of ideas of FontaineMessing). In: Algebraic Geometry, Sendai, 1985, (Adv. Stud. in Pure Math. 10), pp. 207-251, Tokyo, Kinokuniya, 1987

[K2] Kato, K.: Semi-stable reduction and $p$-adic étale cohomology. In: Périodes p-adiques, Séminaire de Bures, 1988, (Astérisuque 223), pp. 269-293, Marseille, Soc. Math. France, 1994

[KCT] Kato, K.: A Hasse principle for two-dimensional global fields. (with an appendix by Colliot-Thélène, J.-L.), J. Reine Angew. Math. 366, 142-183 (1986)

[KS] Kato, K., Saito, S.: Unramified class field theory of arithmetic surfaces. Ann. of Math. 118, 241-275 (1983)

[KL] Katz, N., Lang, S.: Finiteness theorem for higher geometric class field theory. Enseign. Math. 27, 285-319 (1981)

[Ku] Kurihara, M.: A note on $p$-adic étale cohomology. Proc. Japan Acad. Ser. A 63, 275-278 (1987)

[La1] Langer, A.: Selmer groups and torsion zero cycles on the self-product of a semistable elliptic curve. Doc. Math. 2, 47-59 (1997)

[La2] Langer, A.: 0-cycles on the elliptic modular surface of level 4. Tohoku Math. J. 50, 315-360 (1998)

[La1] Langer, A.: Local points of motives in semistable reduction. Compositio Math. 116, 189-217 (1999)

[La2] Langer, A.: Finiteness of torsion in the codimension-two Chow group: an axiomatic approach. In: Gordon, B. B., Lewis, J. D., Müller-Stach, S., Saito, S., Yui, N. (eds.) The Arithemtic and Geometry of Algebraic Cycles, Banff, 1988, (NATO Adv. Sci. Inst. Ser. C Math. Phys. Sci. 548), pp. 277-284, Dordrecht, Kluwer, 2000

[LS] Langer A., Saito, S.: Torsion zero-cycles on the self-product of a modular elliptic curve. Duke Math. J. 85, 315-357 (1996)

[Le1] Levine, M.: Techniques of localization in the theory of algebraic cycles. J. Algebraic Geom. 10, 299-363 (2001)

[Le2] Levine, M.: $K$-theory and motivic cohomology of schemes. preprint, 1999

[Li1] Lichtenbaum, S.: Duality theorems for curves over $p$-adic fields. Invent. Math. 7, 120-136 (1969) 
[Li2] Lichtenbaum, S.: Values of zeta functions at non-negative integers. In: Jager, H. (ed.) Number Theory, Noordwijkerhout, 1983, (Lecture Notes in Math. 1068), pp. 127-138, Berlin, Springer, 1984

[Ma] Mazur, B.: Notes on étale cohomology of number fields. Ann. Sci. École Norm. Sup. (4) 6, 521-552 (1973)

[MS] Merkur'ev, A. S., Suslin, A. A.: $K$-cohomology of Severi-Brauer varieties and the norm residue homomorphism. Math. USSR Izv. 21, 307-341 (1983)

[Mi1] Milne, J. S.: Duality in flat cohomology of a surface. Ann. Sci. École Norm. Sup. (4) 9, 171-202 (1976)

[Mi2] Milne, J. S.: Arithmetic Duality Theorems. (Perspectives in Math. 1), Boston, Academic Press, 1986

[Ne] Nekováŕ, J.: p-adic Abel-Jacobi maps and p-adic height pairings. In: Gordon, B. B., Lewis, J. D., Müller-Stach, S., Saito, S., Yui, N. (eds.) The Arithemtic and Geometry of Algebraic Cycles, Banff, 1998, (CRM Proc. Lecture Notes 24), pp. 367-379, Providence, Amer. Math. Soc., 2000

[O] Otsubo, N.: Selmer groups and zero-cycles on the Fermat quartic surface. J. Reine Angew. Math. 525, 113-146 (2000)

[PS] Parimala R., Suresh, V.: Zero-cycles on quadratic fibrations: Finiteness theorems and the cycle map. Invent. Math. 122, 83-117 (1995)

[Sa1] Saito, S.: Unramified class field theory of arithmetical schemes. Ann. of Math. 1985, 251-281 (1985)

[Sa2] Saito, S.: Arithmetic on two-dimensional local rings. Invent. Math. 85, 379-414 (1986)

[Sa3] Saito, S.: Some observations on motivic cohomologies of arithmetic schemes. Invent. Math. 98, 371-414 (1989)

[Sa4] Saito, S.: On the cycle map for torsion algebraic cycles of codimension two. Invent. Math. 106, 443-460 (1991)

[SaSa] Saito, S., Sato, K.: Cycle class maps for arithmetic schemes (preliminary version). preprint, 2004

[SaSu] Saito, S., Sujatha, R.: A finiteness theorem for cohomology of surfaces over $p$-adic fields and an application to Witt groups. In: Jacob, B., Rosenberg, A. (eds.) Algebraic K-Theory and Algebraic Geometry: Connections with quadratic forms and division algebras, Santa Barbara, 1992, (Proc. of Sympos. Pure Math. 58, Part 2), pp. 403-416, Providence, Amer. Math. Soc., 1995

[Sal] Salberger, P.: Torsion cycles of codimension two and $\ell$-adic realizations of motivic cohomology. In: David, S. (ed.) Séminaire de Thórie des Nombres 1991/92, (Progr. Math. 116), pp. 247-277, Boston, Birkhäuser, 1993 
[Sat1] Sato, K.: Injectivity of the torsion cycle map of codimension two of varieties over $p$-adic fields with semi-stable reduction. J. Reine Angew. Math. 501, 221-235 (1998)

[Sat2] Sato, K.: Logarithmic Hodge-Witt sheaves on normal crossing varieties. preprint, 2004, available at http://www.math.uiuc.edu/K-theory/, $\mathrm{n}^{\circ} 683$

[Sat3] Sato, K.: p-adic étale Tate twists and arithmetic duality. preprint, 2004

[Sch] Schneider, P.: p-adic point of motives. In: Jannsen, U. (ed.) Motives, (Proc. Symp. Pure Math. 55-II), pp. 225-249, Providence, Amer. Math. Soc., 1994

[Scho] Schoen, C.: An integral anolog of the Tate conjecture for onedimensional cycles on varieties over fintie fields. Math. Ann. 311, 493500 (1998)

[Sp] Spiess, M.: On indecomposable elements of $K_{1}$ of a product of elliptic curves. $K$-Theory 17, 363-383 (1999)

[Su] Suresh, V.: Zero cycles on conic fibrations and a conjecture of Bloch. $K$-Theory 10, 597-610 (1996)

[Th] Thomason, R. W.: Absolute cohomological purity. Bull. Soc. Math. France 112, 397-406 (1984)

[Ts1] Tsuji, T.: p-adic étale cohomology and crystalline cohomology in the semi-stable reduction case. Invent. Math. 137, 233-411 (1999)

[Ts2] Tsuji, T.: On $p$-adic nearby cycles of log smooth families. Bull. Soc. Math. France 128, 529-575 (2000)

[Ya] Yamazaki, T.: Formal Chow groups, $p$-divisible groups, and syntomic cohomology. Duke Math. J. 102, 359-390 (2000)

[SGA4] Grothendieck, A., Artin, M., Verdier, J.-L., with Deligne, P., SaintDonat, B.: Théorie des Topos et Cohomologie Étale des Schémas. (Lecture Notes in Math. 269, 270, 305), Berlin, Springer, 1972-73

This article is available at http://intlpress.com/HHA/v7/n3/a9/

Kanetomo Sato kanetomo@math.nagoya-u.ac.jp

Graduate school of Mathematics,

Nagoya University,

Furo-cho, Chikusa-ku,

Nagoya 464-8602,

Japan 